\title{
Randomized Virtual Scanning Technique for Road Network
}

\author{
Bharat Singh \\ M.Tech, Department of C.S \\ Govt. Engg. College, Ajmer \\ (Rajasthan), India
}

\author{
Ravinder Singh \\ M.Tech, Department of I.T \\ Govt. Engg. College, Ajmer \\ (Rajasthan), India
}

\author{
Pramod Singh Rathore \\ M.Tech, Department of C.S \\ Govt. Engg. College, Ajmer \\ (Rajasthan), India
}

\begin{abstract}
In this paper proposes a Randomized Virtual Scanning Technique to optimize the performance for the road network surveillance. The Sensor it may be scan the area of road network. The road network is show as a graph. So it may scan the area through genetic algorithm. The main focus is to improve the energy and performance of the sensor scanning process. The sensing programming rule plays a job for target intrusion detection, utilizing the distinctive options of road networks. Sensor it may be scan alternate and sequence node so that's why $100 \%$ target detection guaranty for w seconds (i.e single sensor takes time to scan the area) along road segments, creating waves of sensing activities, called randomized virtual scanning. Here they present a scan-based algorithm, which improves further energy efficiency or performance of surveillance in road networks.
\end{abstract}

\section{Keywords}

Sensor, Road Network, Randomized Virtual Scanning, Surveillance, Detection, Protection, Scheduling.

\section{INTRODUCTION}

Wireless sensor network (WSN) is nowadays being applied in many different civilian applications like vehicle tracking; habitat monitoring, forest surveillance, earthquake observation, biomedical or health care applications and building surveillance.WSN consist of four main components: A radio, a processor, sensors and battery. A WSN is formed by densely deployed sensor nodes in an application area. In most deployments, the sensor nodes have self-organizing capabilities, to form an appropriate structure in order to collaboratively perform a particular task.

The number of sensors in the WSN is to be providing sufficient sensing coverage and network connectivity. In the roadways are advantage areas for military surveillance and operations. The detection of targets, there are many entrance point and protection point shown in graph where entering from entrance node, before they reach one of protection node. In the field of battle state of affairs awareness needs each entrance nodes and protections nodes to be appointed and modify on demand for quick military maneuver inside a road network.
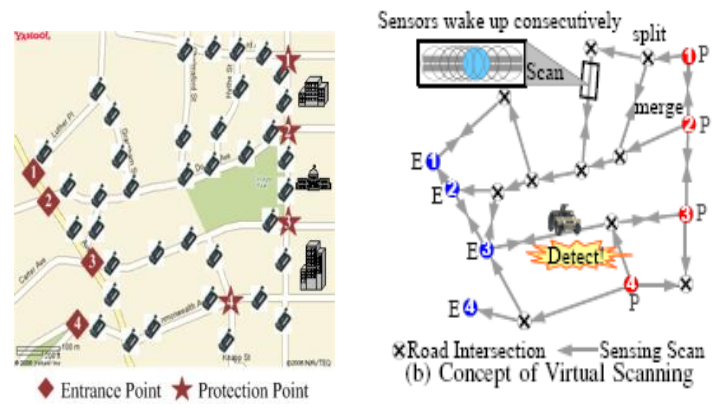

Fig1:(a) Road Network (b)Concept Of Virtual Scanning

The randomized virtual scan of the alternative direction (i.e., from the entrance node purpose to the protection node) can't guarantee target intrusion detection, if a really quick target enters right once the start of the network wide silent time. Sensors wake up one by one but alternate and sequence node (sensor) also scan that's why $100 \%$ target detection guaranty for w seconds along road segments, creating waves of sensing activities, called randomized virtual scanning. Waves propagate from one (or multiple) protection node $\mathrm{P}$, split at the intersections, and merge on the route until they scan all of the road segments. User study reveals that this scan-based methodology can do considerably higher performance (e.g., 10 time's system lifetime) than duty cycling algorithms. Each sensor has constant level of energy that's consumed at constant rate for the sensor's input and sensing operations.

\section{RELATED WORK}

In this paper is to select each sensor's sensing schedule in order to maximize the lifetime of a sensor network, while guarantee that all intruding targets are detected before they reach protection nodes. In this section explain the basic idea of Randomized virtual scanning, using one road segment.

\subsection{Randomized Virtual Scanning for road}

They are representing sensing the coverage using a linear sensor network model as show in Fig. 2 a pair of wherever $n$ sensors square measure linearly placed. That the $\mathrm{n}$ device is placed on the road section rely on the coverage additional space to scan. Every sensor includes a conservative sensing circle of radius $\mathrm{C}_{\text {cover }}$ that is long enough to hide the dimension of the road. Left finish of a road section be the entrance node $\mathrm{E}$ of target and right finish of the road section be the protection node. The period of time of the network is $n$ * life.

Suppose $\mathrm{w}$ is the average detection time to detect the intrusion. So that the sensor can reliably detect a target over 
multiple sampling or $\mathrm{v}_{\text {speed }}$ is a maximum target speed. There are three techniques for scanning for the road network.

\section{Comparisons between all the technique :-}

Always-Awake: - Where sensors are active all the time.

Duty Cycling: - All sensors in the road segment can sleep together for $1 / \mathrm{v}$ seconds but one sensor that time active but it not defines which sensor is active that time and this is defined as silent time of the road network. After that silent time, all nodes wake up simultaneously for during detection.

Virtual Scanning :- In the virtual scanning all the sensors sleep for $1 / \mathrm{v}$ seconds, and it may start the scanning the sensors one by one for working time $\mathrm{w}$ from the right side sensor $\mathrm{s}_{1}$ to left side sensor $\mathrm{s}_{\mathrm{n}}$.

Based on the all the technique they propose a new approach for the randomized virtual scanning. When sensor it may scan same as virtual scanning but they change the scanning process for alter net and reverse so the performance of the scanning is improve and this waves of sensing activities guarantees the detection and allows the additional sleeping time for individual sensors.

So it should compare with duty cycling and virtual scan methodology, this is often be further sleep time is obtained by the very fact that every one sensors however one can sleep throughout the scan time. The direction of a randomized virtual scan of the alternative direction cannot guarantee target intrusion detection, if a really quick target enters right when the start of the network

\subsection{Analytical network lifetime comparison}

It may compares analytically the network lifetime among the all this techniques Always-Awake, Duty Cycling, Virtual Scanning and Randomized Virtual Scanning methods.

The Always-Awake approach, the network lifetime is the same as sensor life, because sensors work continuously without sleeping.

Duty cycling approach, the network lifetime is the number of periods multiplied by the length of the periods.

Virtual scanning approach, the network lifetime is the number of periods multiplied by the period length. Number of period is the sum of the scan time nw and silent time

Randomized virtual scanning approach, the network lifetime is the number of periods multiplied by the period length. Number of period is the sum of the scan time 2 multiplied scan time nw and silent time.

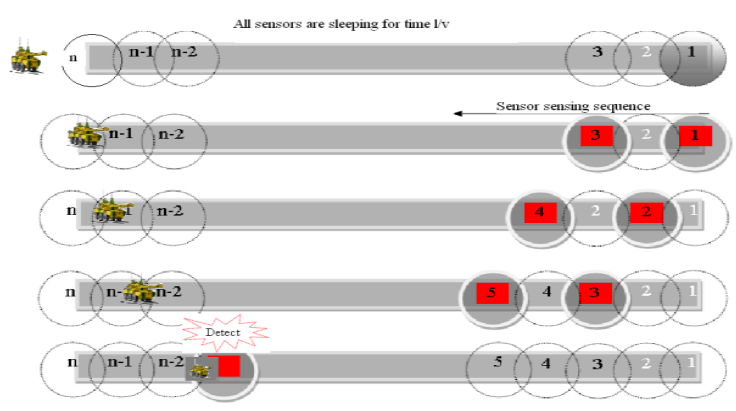

Fig. 2 Sensor Network Model for Road Segment

\subsubsection{Approach}

There are several entrance node and protection node which show fig.1 (b) , it may be define the process of the scanning graph. As from the above graph user draw a Scanning process in fig 3 as a sub graph. Here user defines the process of scanning:

Step 1: It may select the one single entrance point and one protection (fig. 3) on the multiple points for entrance or protection points in the graph.

$$
\begin{aligned}
& \mathrm{V}_{1 \rightarrow} \text { Protection node (source point) } \\
& \mathrm{V}_{7 \rightarrow} \text { Entrance node (destination point) }
\end{aligned}
$$

Step 2 : figure 3 shows the scanning process for randomly.

Step 3 : The scanning process as follows, it may start from right side of protection point the sensor active first for $\mathrm{v}_{1}$ and $\mathrm{v}_{3}$ at a time whereas all other are in sleeping mode.

Step 4 : The scanning time for each sensor is w (fixed time) and for scanning complete nodes, it just move on the active for $\mathrm{v}_{2}$ and $\mathrm{v}_{4}$ sensor node then after $\mathrm{v}_{3}$ and $\mathrm{v}_{5}$ sensor active. Two sensors active at a time so the target is detect $100 \%$, so it may improve the performance.

So it may improve the performance of randomized virtual scan method.

- In the surveillance for road networks, the randomized scan-based sensing algorithm (RVISA) opens a promising direction.

- RVISA takes advantages of road network's characteristics for a maximum sleeping time.

* Vehicles move along the roadways whose maps are available.

* Contributions

- Energy-efficient Sensing Schedule for Road Networks 

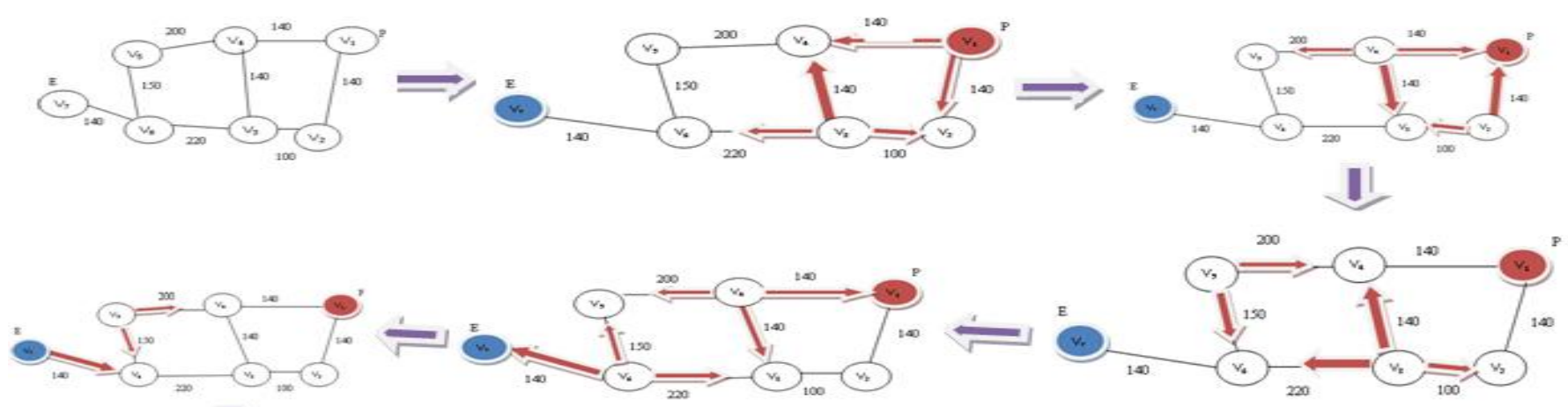

Fig 3: Scanning Process

* RVISA uses the optimal sleeping time for an arbitrary road network.

RVISA can control the trade-off between the network lifetime and average detection time. Here are some notation which use in the below algorithm:

\begin{tabular}{|c|c|}
\hline $\mathrm{E}_{\mathrm{p}}$ & $\begin{array}{l}\text { A set of vertices for the entrance node of the } \\
\text { road network }\end{array}$ \\
\hline $\mathrm{v}_{\text {speed }}$ & A maximum speed of target: unit is $[\mathrm{m} / \mathrm{sec}]$ \\
\hline $\mathrm{C}_{\text {cover }}$ & $\begin{array}{l}\text { The length of rectangle covered by one sensor: } \\
\text { unit is }[\mathrm{m}]\end{array}$ \\
\hline $\mathrm{E}_{\text {on }}$ & Turn-on energy of sensor: unit is $[\mathrm{J}]$ \\
\hline $\mathrm{S}_{\text {power }}$ & Sensing power: unit is [watts] \\
\hline $\mathrm{W}$ & Average Detection time [set by default ] \\
\hline M & $\begin{array}{l}\text { A matrix that has each sensor's scheduling in } \\
\text { information }\end{array}$ \\
\hline $\mathrm{G}$ & It denote the Graph for the road network. \\
\hline
\end{tabular}

Algorithm: Sensor Schedule $\left(\mathrm{E}_{\mathrm{p}}, \mathrm{w}, \mathrm{v}_{\text {speed }}, \mathrm{C}_{\text {cover }}, \mathrm{E}_{\mathrm{on}}, \mathrm{S}_{\text {power }}\right)$ Step 1: \{Function description:

(i) Determine the sleeping time $\mathrm{s}$ for the shortest path from the entrance node towards the protection node that satisfies the required $\mathrm{w}$,

(ii) Find the set of sensors $\mathrm{S}$ nearest to the next nearest node that start the scanning simultaneously after the sleeping period $\mathrm{s}$, and

(iii) Determine the working matrix $\mathrm{M}$ containing the acceptable working amount of every detector that participates within the scanning. \}
Step 2: $1 \leftarrow \mathrm{w} \cdot 2 \mathrm{v}_{\text {speed }}\left\{\mathrm{w}=1 / 2 \mathrm{v}_{\text {speed }}\right\}$

Step 3: if $\mathrm{E}_{\text {on }}<\mathrm{C}_{\text {cover }} \cdot \mathrm{S}_{\text {power }} / \mathrm{v}$ then

$$
\mathrm{s} \leftarrow \mathrm{l} / \mathrm{v} \text { \{compute sleeping time } \mathrm{s} \text { \} }
$$

else

$\mathrm{s} \leftarrow 0$ \{sleeping time $\mathrm{s}$ is set to zero\}

end if

Step 4: $\mathrm{S} \leftarrow$ Find Starting Points $\left(\mathrm{G}, \mathrm{E}_{\mathrm{p}}, \mathrm{l}\right)$

\{find the set of vertices $\mathrm{S}$ consisting of starting

points on $\mathrm{G}$ to satisfy the w

Step 5: $\mathrm{M} \leftarrow$ Compute Working Matrix $\left(G, E_{p}, S\right)$

\{compute the working time matrix $\mathrm{M}$ whose entry value is working time of sensors on the corresponding edge $\}$

Step 6: End

\section{EXPERIMENTAL EVALUATION}

In this case, they present experimental evaluations to compare the above approach. We use synthetic networks dataset. Experiments are conducted to on different parameter and their impact can be seen by the graph.

There are several methods for Scanning, Duty Cycling, Virtual Scanning and Always- Awake can give surety for the detection of targets. There is several methods and technique use to improve the network lifetime and performance to detect of target. The Always-awake technique sleeping time is zero and duty cycling method is use the more sleeping time compare to Always-awake method. All the methods have different sleeping time so it may define all methods. Virtual Scanning has the longest sleeping time and hence the longest network lifetime. For example, randomized Virtual Scanning sustains 3.2 hours, compared with Virtual Scanning 28.2 hours, compared with 1.4 hours in Duty athletics and five. 4 minutes in Always-Awake. This is often attributable to the numerous energy savings throughout the scanning method. 
Randomized Virtual Scanning lets the sleeping time degrade gradually.

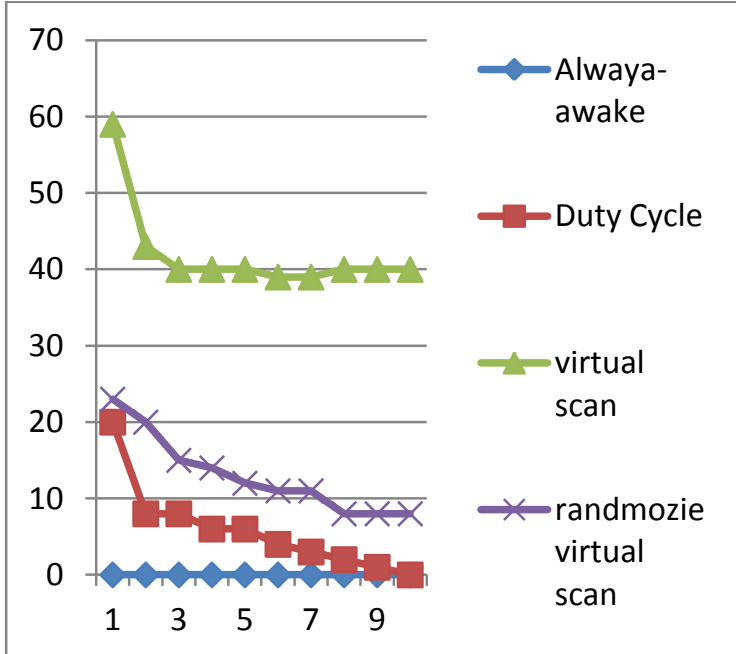

Fig. 4 Graph representation for sensing

\section{CONCLUSION}

In the road network, this work introduces RVISA based on the concept of virtual scanning. RVISA sensing waves detects along the road network before they reach the protection nodes. We propose the scanning method for scan the area to detect the vehicles enter the area and improve the performance. We believe that this work opens a promising detection of road network surveillance.

\section{ACKNOWLEDGEMENTS}

User wish to thank Mr. Ravinder Singh, Asst. Professor, Computer Department whose guidance, teaching and certain suggestion provide me the timely valuable input which enhanced my knowledge

\section{REFERENCES}

[1] Z. Abrams, A. Goel, and S. Plotkin, ACM/IEEE, 2004. "Set K-Cover Algorithms for Energy Efficient Monitoring in Wireless Sensor Networks,".

[2] M. Cardei, M. T. Thai, Y. Li, and W. Wu, 2005, "EnergyEfficient Target Coverage in Wireless Sensor Networks," in IEEE. INFOCOM,

[3] S. Kumar, T. H. Lai, and J. Balogh., 2011, "On KCoverage in a Mostly Sleeping Sensor Network," in MOBICOM. ACM,.

[4] D. Tian and N. Georganas, May 2011, "A Node Scheduling Scheme for Energy Conservation in Large Wireless Sensor Networks," Wireless Communications and Mobile Computing Journal,

[5] J. Jeong, Y. Gu, T. He, and D. Du, April 2009, "VISA: Virtual Scanning Algorithm for Dynamic Protection of Road Networks," in Proc. of 28th IEEE Conference on Computer Communications (INFOCOM 09), Rio de Janeiro, Brazil.

[6] M. Mar 'oti, B. Kusy, G. Simon, and 'Akos L'edeczi, , Nov. 2004, "The Flooding Time Synchronization Protocol," in SENSYS. Baltimore, Maryland, USA: ACM.

[7] L. Lazos, R. Poovendran, and J. A. Ritcey, Analytic March 2009, evaluation of target detection in eterogeneous wireless sensor networks," ACM Trans.Sensor Networks, vol. 5, no. 2, pp. 1-38.

[8] J. Hwang, T. He, and Y. Kim, Nov. 2007, "Exploring InSitu Sensing Irregularity in Wireless Sensor Networks," in SENSYS. ACM, , pp. 289-303.

[9] Y. Gu and T. He, Nov. 2007, "Data Forwarding in Extremely Low Duty-Cycle Sensor Networks with Unreliable Communication Links," in SENSYS. Sydney, Australia: ACM, pp. 321-334.

[10] G. Lu, N. Sadagopan, B. Krishnamachari, and A. Goel, 2005 "Delay Efficient Sleep Scheduling in Wireless Sensor Networks," in INFOCOM. IEEE 